\title{
Circulation: Cardiovascular Quality and Outcomes
}

\section{ORIGINALARTICLE}

\section{Characteristics and Quality of National Cardiac Registries: A Systematic Review}

\author{
Luke P. Dawson®, MBBS, MPH; Sinjini Biswas, MBBS, PhD; Jeffrey Lefkovits, MBBS; Dion Stub, MBBS, PhD; \\ Luke Burchill, MBBS, PhD; Sue M. Evans, PhD; Christopher Reid®D, BA DipEd, PhD; David Eccleston, MBBS, MMedSci
}

BACKGROUND: National cardiac registries are increasingly used for informing health policy, improving the quality and costeffectiveness of patient care, clinical research, and monitoring the safety of novel treatments. However, the quality of registries is variable. We aimed to assess the characteristics and quality of national cardiac registries across all subspecialties of cardiac care.

METHODS: Publications relating to national cardiac registries across six cardiac subspecialty domains were identified by searching MEDLINE and the Google advanced search function with 26438 citations and 4812 full-text articles reviewed.

RESULTS: A total of 155 registries, representing 49 countries, were included in the study. Of these, 45 related to coronary disease or percutaneous coronary intervention, 28 related to devices, arrhythmia, and electrophysiology, 24 related to heart failure, transplant, and mechanical support, 21 related to structural heart disease, 21 related to congenital heart disease, and 16 related to cardiac surgery. Enrollment was procedure-based in 60\% and disease-based in 40\%. A total of 73.10 million patients were estimated to have been enrolled in cardiac registries. Quality scoring was performed using a validated registry grading system, with registries performing best in the use of explicit variable definitions and worst in assessment of data reliability. Higher quality scores were associated with government funding, mandated enrollment, linkage to other registries, and outcome risk adjustment. Quality scores and number of registries within a country were positively correlated with each other and with measures of national economic output, health expenditure, and urbanization.

CONCLUSIONS: There has been remarkable growth in the uptake of national cardiac registries across the last few decades. However, the quality of processes used to ensure data completeness and accuracy remain variable and few countries have integrated registries covering multiple subspecialty domains. Clinicians, funders, and health policymakers should be encouraged to focus on the range, quality, and integration of these registries.

REGISTRATION: URL: https://www.crd.york.ac.uk/prospero; Unique identifier: CRD42020204224.

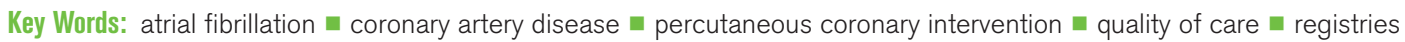

\section{See Editorial by Murugiah and Masoudi}

0 ver the past few decades, there has been expansive growth in the range and number of clinical registries. Clinical registries can be used to inform health policy, improve the quality and cost-effectiveness of patient care, allow for access to data for clinical research, and assist in monitoring the uptake and safety of novel treatments and procedures. ${ }^{1-3}$ Although not universal, in some cases registry initiation has been associated with improvements in patient outcomes and mortality. ${ }^{4-6}$ The field of cardiology has been at the forefront of registry development and many countries have developed highly complex networks to collect data on the full spectrum of cardiac procedures and disease presentations.

Correspondence to: David Eccleston, MBBS, MMedSci, Department of Cardiology, The Royal Melbourne Hospital, 300 Grattan St, Parkville, Victoria 3050 , Australia. Email david.eccleston@mh.org.au

The Data Supplement is available at https://www.ahajournals.org/doi/suppl/10.1161/CIRCOUTCOMES.121.007963.

For Sources of Funding and Disclosures, see page 994.

(C) 2021 American Heart Association, Inc.

Circulation: Cardiovascular Quality and Outcomes is available at http://www.ahajournals.org/journal/circoutcomes 


\section{WHAT IS KNOWN}

- Clinical registries are used for a variety of purposes and can be useful in research, quality of care, and monitoring safety.

- Data management practices and registry purpose are highly varied.

\section{WHAT THE STUDY ADDS}

- This study quantifies the enormous growth in both number and scale of national cardiac registries over the last 40 years.

- Quality of data management practices and registry enrollment are correlated with measures of national economic output and health expenditure.

- Specific aspects of registry design should be considered when determining a registry's suitability for a given purpose.

\begin{tabular}{|ll|}
\hline Nonstandard Abbreviations and Acronyms \\
\hline PCI & $\begin{array}{l}\text { percutaneous coronary } \\
\text { intervention }\end{array}$ \\
CAD & coronary artery disease \\
GDP & gross domestic product \\
\hline
\end{tabular}

Just as cardiac registries are extremely broad in scope of subspecialties covered, the data management and quality assurance methods are similarly varied. More developed national registries cover multiple subspecialties, have extensive linkage with other population-based databases, and comprehensive data auditing systems to ensure data completeness and accuracy. ${ }^{3,7-11}$ Several national cardiac registries are mandated by the government as part of procedural or device reimbursement. ${ }^{12,13}$ Conversely, less well-developed registries may simply document procedures with minimal or no outcome data using processes for data collection that are highly susceptible to bias. The ability of a registry to achieve its goals of informing policy, improving quality of care, and generating clinical research is dependent on the quality of the registry.

Several studies have analyzed registry characteristics for several topics in cardiology, such as percutaneous coronary intervention $(\mathrm{PCl}),{ }^{1}$ cardiac implant devices, ${ }^{14}$ and transcatheter aortic valve implantations. ${ }^{15}$ However, no studies have analyzed cardiac registries across the whole cardiac specialty-the aim of this study. National registries were selected for this purpose given they are likely to be the largest, best resourced, with the most robust governance structure, and be more representative in comparison to regional registries. Similarly, national registries may be less subject to biases introduced by regional practice trends and local socioeconomic and cultural factors. Furthermore, it is useful to score and assess the quality of national registries as they are the most likely to be used to inform governments, health providers, researchers, and health economists in their work. Therefore, the purpose of this systematic review was to summarize the characteristics of national cardiac registries across all subspecialties of cardiac care. Furthermore, we aimed to perform a quality assessment of each registry's enrollment and data processes using a well-validated clinical registry quality score.

\section{METHODS}

This review has been performed in compliance with the Preferred Reporting Items for Systematic Reviews and Metaanalyses statement, ${ }^{16}$ and the protocol was registered in the Prospective Register of Systematic Reviews. All data and supporting materials have been provided with the published article and Data Supplement. The search protocol was developed by two authors (L.D. and D.E.) and objectives, search methods, and registry inclusion criteria were prespecified before the commencement of the study. Cardiac registries were classified into 6 categories: those capturing: (1) congenital heart disease; (2) structural heart disease; (3) cardiac surgery; (4) devices, arrhythmia, and electrophysiology; (5) coronary artery disease (CAD), coronary angiography, and $\mathrm{PCl}$; and (6) heart failure, cardiomyopathies, mechanical supports, and transplant. This study did not require approval by an institutional ethical review board as no individual patient data were included and data regarding registries were publicly available.

\section{Search Strategy}

Two search strategies were used to identify publications relating to cardiac registries among six subspecialty domains: (1) A MEDLINE search for publications relating to cardiac registries across the 6 defined cardiac subspecialties, from inception until August 2019, limited to humans (this search was updated in January 2021 to include the period from August 2019 to December 2020); (2) an internet search of webpages using the Google Advanced search function, whereby the terms cardiac and registry were combined with the names of all United Nations member countries to identify webpages, reports, abstracts and publications not identified in the MEDLINE search. The searches were not limited by language and non-English publications were assessed for eligibility using Google translate. The specific search strategy and medical subject headings terms used for the MEDLINE search are presented in Table I in the Data Supplement.

\section{Eligibility Criteria}

Study inclusion was limited to national registries with a countrywide system for data collection on that cardiac domain that were actively collecting data at the time of search, and had reports, journal publications, abstracts, or online material that could be accessed and assessed by reviewers. Studies 
that were regional or international were excluded, other than those which were binational in the same geographic region, for example, Australia/New Zealand; Sweden/lceland; United Kingdom/Ireland. Studies were excluded if they were no longer actively collecting data at the time of assessment, or if eligibility for study inclusion could not be adequately assessed due to very limited published material and no contact information (studies excluded with reasons are summarized in Table VI in the Data Supplement). Cardiac arrest registries were not included in the review to avoid including registries that related to multiple specialties (ie, emergency medicine, intensive care medicine) rather than being exclusively related to cardiology.

\section{Data Extraction and Quality Scoring}

Data were extracted for each eligible registry using a standardized study form to determine: (1) registry name and geographic location; (2) year of registry commencement; (3) subspecialty domains covered by the registry, including procedures or disease processes entered; (4) most recently published total number of patients included in the registry, and year of publication; (5) outcome timepoints collected; (6) whether annual reports are published, risk adjustment performed, linkage to other registries, government-mandated enrollment, and funding sources; and (7) the components of the composite quality score described below. Enrollment of participants was classified as either disease or procedural based. Disease-based enrollment included heart failure, cardiomyopathy, atrial fibrillation, CAD, acute coronary syndromes, and congenital heart disease registries, whereas procedural-based registries included coronary angiography, $\mathrm{PCl}$, surgery, intervention, device, ablation, and transplant. Attributes of registries were captured directly from journal articles, abstracts, annual reports, and registry webpages or directly from registry personnel using a study form (Figure I in the Data Supplement). An attempt was made to contact each registry via the email listed on the registry webpage, or, if not available, the corresponding author email listed on the registry's most recent publication. Contact details were identified for $95 \%$ of registries, and a response was received for $17 \%$.

Data quality of included registries was assessed using a composite score for clinical quality registries adapted, with permission, from a validated grading system published by $\mathrm{Md}$ Emdadul Hoque et al in 2017.17 This grading system calculates a composite score out of a maximum of twenty-four points assessing enrollment of eligible population, reliability of data coding, methods of data validation, independence of observers to primary outcomes, completeness of data, and use of explicit variable definitions (Table).

\section{National Gross Domestic Product and Health Expenditure Data}

To determine country-based factors associated with the number and quality of national cardiac registries, we accessed publicly available World Bank data regarding country population, annual gross domestic product (GDP), annual GDP per capita, healthcare expenditure per capita, healthcare expenditure as a percentage of GDP, and urban population (\% of total population). ${ }^{18}$

\section{Statistical Analysis}

Estimated patient numbers were determined from registry correspondence or calculated using linear growth from registry inception until current year using the most recent publication year and patient number at that time as a single data point. Quality scores were presented as median (interquartile range [IOR]) with comparison between groups using the MannWhitney $U$ test (or the chi-square test for individual components). To determine correlation between the number of national cardiac registries and quality scores we included registry size, inception year, country population, annual GDP, annual GDP per capita, healthcare expenditure, healthcare expenditure as a percentage of GDP, and urban population (\% of total population) in a correlation matrix using Spearman's correlation coefficient. Statistical analysis was conducted using Stata 14.2 for Mac (College Station, TX) and R version 3.6.2 (The R Foundation for Statistical Computing, Vienna, Austria) with the ggplot2 visualization package. Mapping was performed using ArcGis (ESRI, Redlands, CA) to summarize registry data by country, including (1) the number of subspecialty domains covered; (2) the median registry quality score; and (3) the total number of patients or procedures entered in registries across all subspecialty domains. All calculated $P$ values were 2 sided, and a $P<0.05$ was considered statistically significant.

\section{Table. Modified Monash University Clinical Quality Registry Grading System}

\begin{tabular}{|c|c|c|}
\hline Component & Criteria & Patients \\
\hline Enrollment & $<80 \%$ or not available & 1 \\
\hline \multirow{3}{*}{$\begin{array}{l}\% \text { patients in registry out of } \\
\text { at-risk population }\end{array}$} & $80 \%-89 \%$ & 2 \\
\hline & $90 \%-97 \%$ & 3 \\
\hline & $>97 \%$ & 4 \\
\hline Reliability & No audit & 1 \\
\hline \multirow{3}{*}{$\begin{array}{l}\text { Interobserver and intraob- } \\
\text { server agreement }\end{array}$} & Kappa<0.5 & 2 \\
\hline & Kappa 0.5-0.8 & 3 \\
\hline & Kappa $>0.8$ & 4 \\
\hline Data validation & No audit & 1 \\
\hline \multirow[t]{3}{*}{ Method of data checks } & Range or consistency & 2 \\
\hline & Range and consistency & 3 \\
\hline & External audit & 4 \\
\hline Outcomes & No outcome & 1 \\
\hline \multirow{3}{*}{$\begin{array}{l}\text { Independence of observers } \\
\text { to outcomes }\end{array}$} & Not independent & 2 \\
\hline & Not blinded & 3 \\
\hline & Independent and blinded & 4 \\
\hline Completeness & $<50 \%$ or not available & 1 \\
\hline \multirow{3}{*}{$\begin{array}{l}\% \text { variables with }>95 \% \text { data } \\
\text { complete }\end{array}$} & $50 \%-70 \%$ & 2 \\
\hline & $80 \%-97 \%$ & 3 \\
\hline & $>97 \%$ & 4 \\
\hline Definitions & $<50 \%$ or not available & 1 \\
\hline \multirow{3}{*}{$\begin{array}{l}\% \text { variables with explicit } \\
\text { definitions }\end{array}$} & $50 \%-70 \%$ & 2 \\
\hline & $80 \%-97 \%$ & 3 \\
\hline & $>97 \%$ & 4 \\
\hline \multicolumn{2}{|l|}{ Total score } & 24 \\
\hline
\end{tabular}

Clinical quality registry score grading system is adapted from Md Emdadul Hoque et al ${ }^{17}$ with permission. Copyright (C)2018, Oxford University Press. 


\section{RESULTS}

The MEDLINE search identified 26438 citations and the Google Advanced search identified a further 25 registries not identified through the MEDLINE search. In total, 4812 full-text articles were screened for relevance, and 155 registries met criteria for inclusion (Figure 1).

\section{Registry Characteristics}

Of the included registries, 45 related to $\mathrm{CAD}$ or $\mathrm{PCl}(19$ acute coronary syndromes or CAD, 11 coronary angiography/ $\mathrm{PCl}, 15 \mathrm{PCl}), 28$ related to devices, arrhythmia, and electrophysiology (2 devices and ablation, 14 devices-only, 8 atrial fibrillation, 4 electrophysiologyonly), 24 related to heart failure, cardiomyopathies, mechanical support and transplant (13 heart failure, 2 transplant, 2 mechanical support, 7 other), 21 related to structural heart disease (6 multiple structural procedures, 13 transcatheter aortic valve implantation, 1 mitra-clip, and 1 other), 21 related to congenital heart disease (14 general, 4 surgical, 2 interventional, and 1 specific disease process), and 16 related to cardiac surgery. In total, there were 93 procedure-based registries (60\%; eg, coronary angiography, $\mathrm{PCl}$, surgery, intervention, device, ablation, transplant), and 62 disease-based registries (40\%; eg, heart failure, cardiomyopathy, atrial fibrillation, $C A D$, acute coronary syndrome, congenital heart disease). Outcomes were limited to in-hospital only in 43 registries (28\%), whereas 100 registries (65\%)

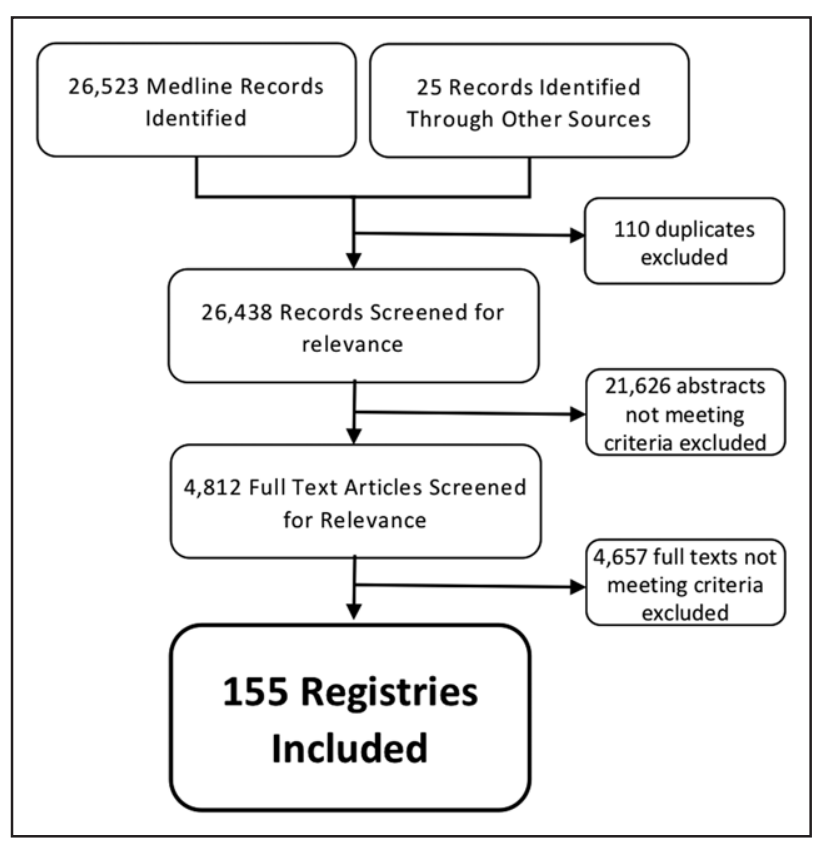

Figure 1. Preferred Reporting Items for Systematic reviews and Meta-Analyses (PRISMA) diagram.

PRISMA diagram summarizing the search strategy used to identify national cardiac registries meeting inclusion criteria. followed participants beyond the in-hospital period, and 12 registries (8\%) did not record outcomes.

Included registries involved 49 countries, with 4 countries covering all six subspecialty domains (Denmark, Sweden, United Kingdom, United States), 3 countries covering 5 domains, 8 countries covering four domains, 7 countries covering three domains, 11 countries covering 2 domains, and 16 countries covering one domain. Integrated national cardiac registries covering multiple subspecialty domains were identified in 10 countries (Denmark, Finland, Iran, Norway, Singapore, Sweden, Iceland, Taiwan, United Kingdom, United States; Table III in the Data Supplement).

Annual reports were completed for 49 registries (32\%) with 28 registries (18\%) using risk adjustment when comparing outcomes between service providers. Government-mandated enrollment for the at-risk population (eg, by linking procedural reimbursement to registry enrollment) was used in 37 registries (24\%). Linkage to other population-based registries, such as the national death register, was used in 48 registries (31\%). Official websites were identified for 80 registries (52\%). Most registries were funded by their relevant cardiac society (45\%) and/or government (37\%). World maps summarizing subspecialty domain coverage, registry quality scores, and patient numbers are presented in Figure 2.

\section{Estimated Patient Numbers}

We estimated that 73.10 million patients have been entered into national cardiac registries, comprising 1.31 million congenital heart disease patients, 11.03 million cardiac surgery patients, 0.33 million structural heart disease patients, 48.34 million CAD or $\mathrm{PCl}$ patients, 9.31 million device and electrophysiology patients, and 2.78 million heart failure patients. Registry numbers and estimated patient growth from 1980 until 2020 separated by subspecialty domain are summarized in Figure 3. A full summary of registry characteristics are presented in Tables III and IV in the Data Supplement.

\section{Quality Scores}

Of a total potential quality score of 24 , the overall median quality score was 15 (IOR, 12-19). Cardiac surgery registries had the highest quality score (median 19, IQR, 10-22), followed by structural heart disease (median 16, IOR, 13-21), CAD and PCl (median 16, IOR 13-19), congenital heart disease (median 14, IQR, 12-20), devices and electrophysiology (median 14, IOR, 12-17), and heart failure (median 13.5, IOR, $11-17)$. In specific components of the quality score, registries performed best in using explicit variable definitions ( $94 \%$ of registries achieving 3 or 4 points), 


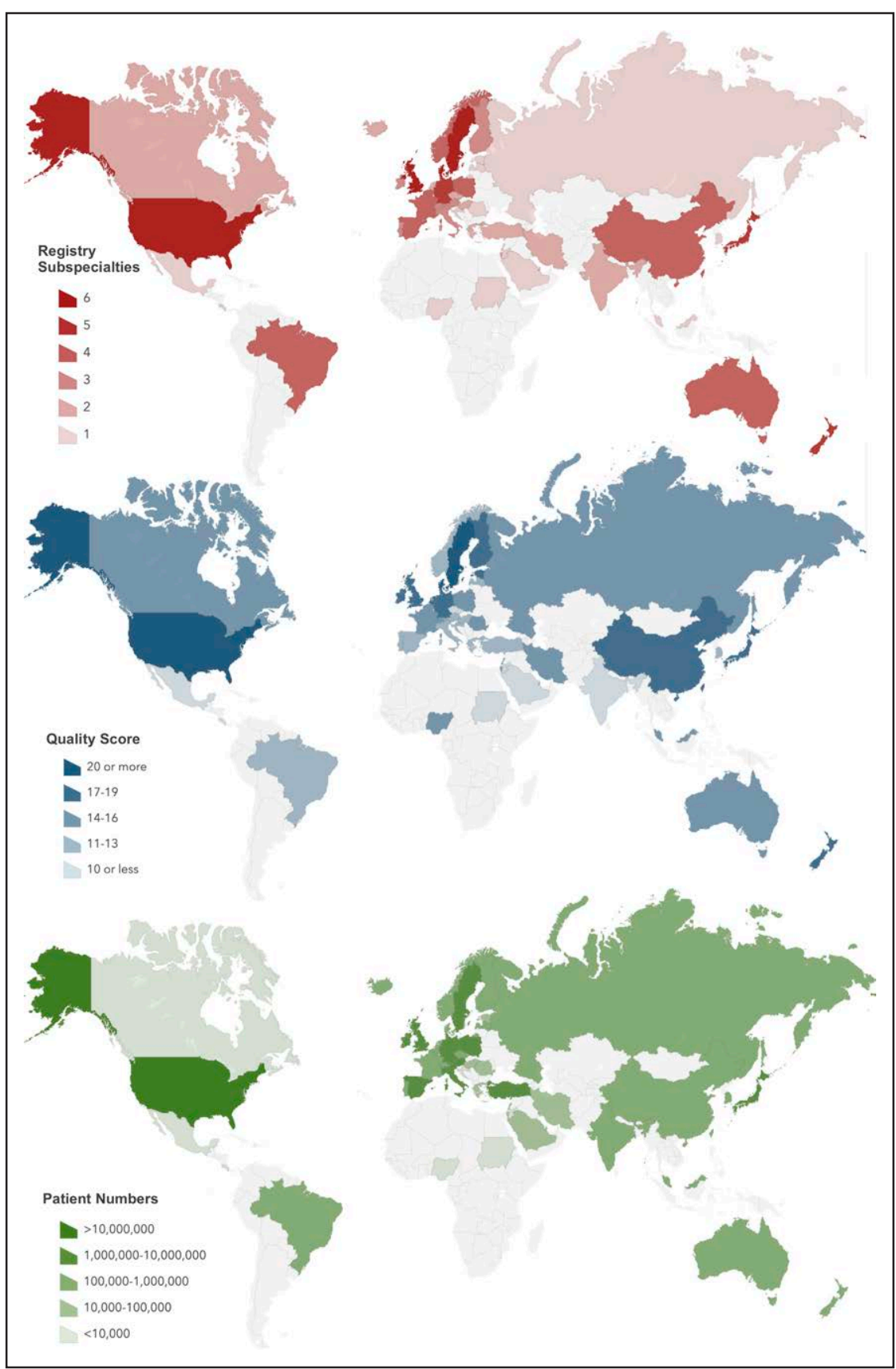

Figure 2. National cardiac registries world map.

World map illustrating number of subspecialty domains covered by country in red, median quality score by country in blue, and estimated number of patients entered into registries by country in green. followed by independent outcome assessment (69\% scoring 3 or 4 points), data validation methods (53\% scoring 3 or 4 points), coverage of at-risk population (49\% scoring 3 or 4 points), data completeness (38\% scoring 3 or 4 points), and assessment of data reliability (27\% scoring 3 or 4 points; Figure 4). Higher quality scores were associated with government funding (median 17, IQR, 13-21 versus median 14, IOR, 11-18, $P<0.01)$, government-mandated enrollment (median 19, IQR, 15-22 versus median 13, IOR, 11$17, P<0.001)$, linkage to other population-based registries (median 19, IQR, 15-21 versus median 13, IQR, $11-17, P<0.001$ ), and the use of outcome risk adjustment in annual reports (median 21.5, IQR, 19-23 versus median 13, IQR, 11-17, $P<0.001$ ). Registries enrolling by disease process performed worse in the at-risk population enrollment component of the quality score (24\% versus $65 \%$ scoring 3 or 4 points, $P<0.01$ ) compared with registries enrolling by procedure. Conversely, procedural-based registries performed worse in the observer independence to outcome assessment component of the score (62\% versus $79 \%$ scoring 3 or 4 points, $P=0.03$ ). Individual components of the quality score for each registry are presented in Table $V$ in the Data Supplement. Furthermore, Figure II in the Data Supplement provides a guide to the degree of importance of each component according to registry purpose. 


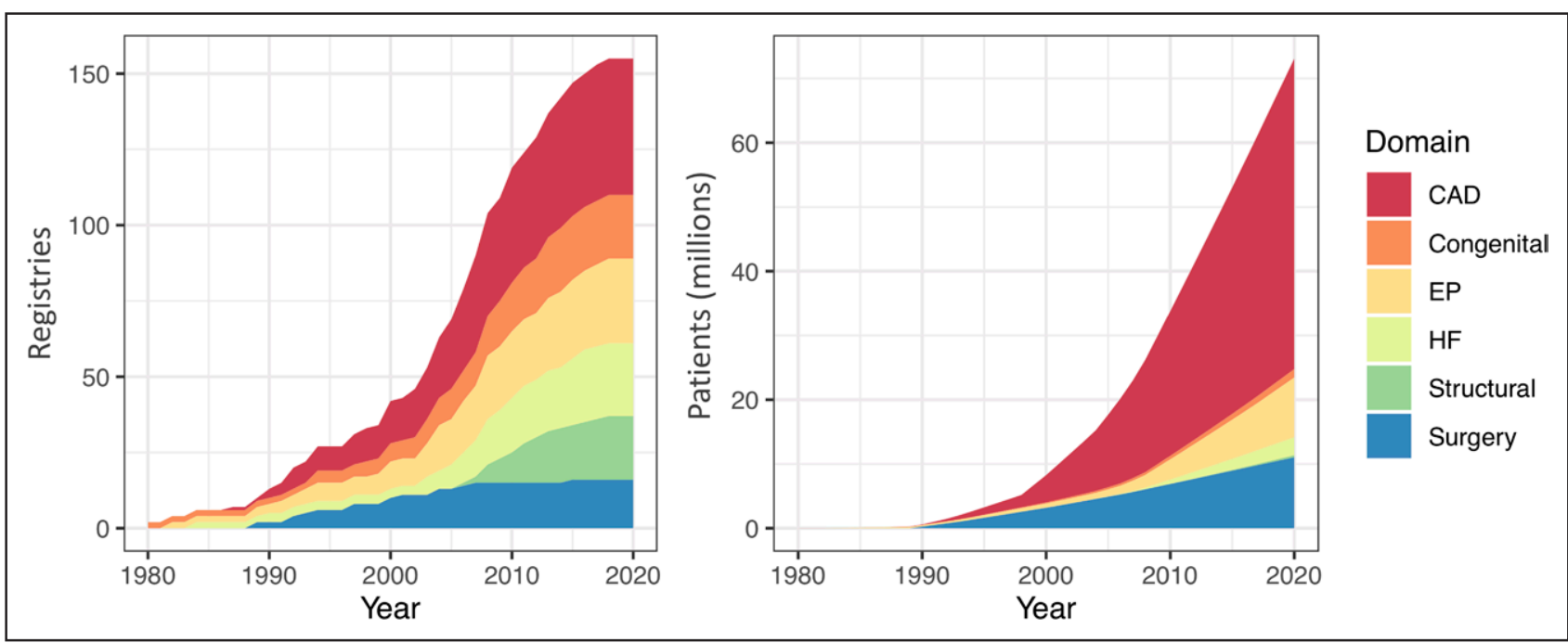

Figure 3. National cardiac registry growth 1980-2020.

Change in number of national cardiac registries and estimated total patients entered into national cardiac registries separated by subspecialty domain from 1980 to 2020 . CAD indicates coronary artery disease; EP, electrophysiology; and HF, heart failure.

\section{Correlates With Registry Number and Quality}

Registry quality scores had a moderate positive correlation with number of subspecialty domains covered by that country, total number of national registries, number of patients in the registry, GDP, GDP per capita, health expenditure, health expenditure as a percentage of GDP, and percentage of urban population (all $P<0.05$; Figure 5). Number of subspecialty domains covered and total number of national registries both had a highly positive correlation with each other, GDP, GDP per capita, health expenditure, and health expenditure as a percentage of GDP (all $P<0.05$ ).

\section{DISCUSSION}

In this systematic review of 155 national cardiac registries, across 49 countries, the major findings are as follows: (1) there has been enormous growth in national cardiac registries over the last four decades with an estimated 73 million patients entered; (2) a minority of

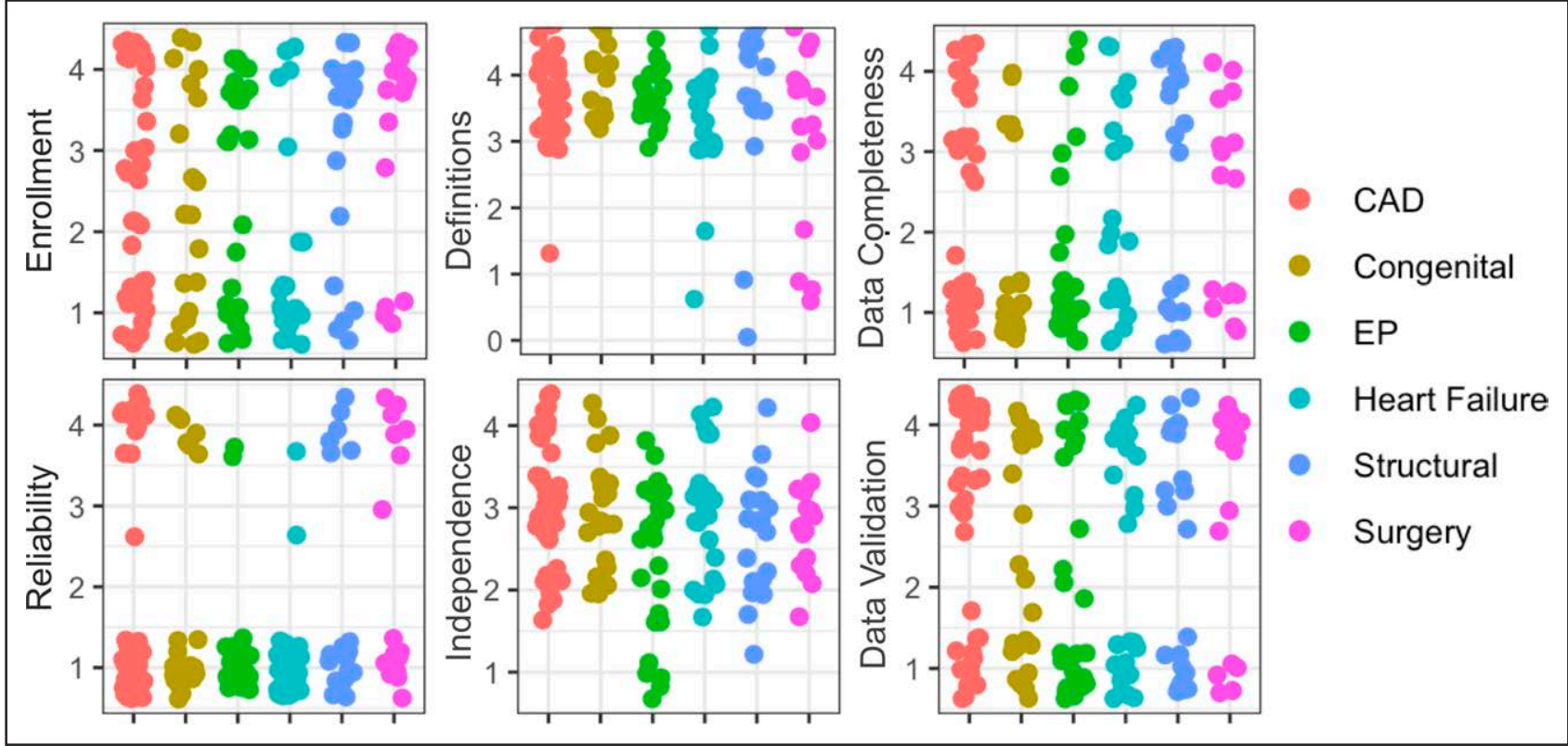

Figure 4. Individual quality score components.

Jitter plot demonstrating individual components of the quality score separated by subspecialty domain, including enrollment of at-risk population, interobserver and intraobserver coding reliability, use of explicit variable definitions, independence of observers from outcomes, data completeness for each variable collected, and methods of data validation (see Table). CAD indicates coronary artery disease and percutaneous coronary intervention; and EP, electrophysiology. 
countries have integrated registries, whereas most have separate registries that are subspecialty specific; (3) quality scores vary, and among individual components, registries performed best at using explicit definitions and outcome assessment and worst at data completeness and reliability; (4) more registries enrolled by procedure rather than disease process, with the former more likely to enroll a higher proportion of the at-risk population; and (5) a country's registry quality scores and number of registries correlated with each other and with measures of national economic output, health expenditure, and urbanization.

The expansive growth in the uptake of national cardiac registries identified in this study is remarkable, but not surprising given the many benefits. There are several types of clinical registries including drug/device registries, research registries, condition or disease registries, and clinical quality registries, with the latter forming the bulk of the national registries identified in this study. The key role of a clinical quality registry is to foster benchmarking and quality assurance across health care areas and facilitate quality improvement in patient care. In cardiology, networks such as the National Institute for Cardiovascular Outcomes Research in the United Kingdom, ${ }^{19}$ the National Cardiovascular Data Registry in the United States, ${ }^{10}$ and the SWEDEHEART (Swedish Web-System for Enhancement and Development of Evidence-Based Care in Heart Disease Evaluated According to Recommended Therapies) in Sweden, ${ }^{9}$ are key examples of registries that achieve this aim, providing annual or quarterly, detailed, risk-adjusted, quality of care reports to health services that can inform local hospital actions. Furthermore, registries may allow novel approaches to unforeseen events, such as the rapid adaption of the American Heart Association Get With The Guidelines registries to begin collecting data relating to coronavirus disease 2019 (COVID-19) cardiovascular outcomes during the COVID-19 pandemic. ${ }^{20}$ Other benefits of registries include informing health policy, facilitating research on large cohorts, regulation of devices and operators, and monitoring the uptake and safety of new treatments and procedures. ${ }^{21}$ The use of registries for benchmarking and regulation has resulted in extensive discussion in recent years regarding what outcomes should be reported/used (eg, mortality versus process measures like readmission), how much risk adjustment is required, and whether public reporting of data is appropriate. Public reporting has been controversial with advocates arguing that patients should have access to data regarding their proposed care, whereas critics raise concerns that high-risk patients may be denied care by risk-averse clinicians. ${ }^{22}$ Improved risk adjustment, better outcome measures, and a shift towards disease-based rather than procedure-based registries have been suggested as potential solutions. ${ }^{23}$

In spite of the many benefits, the value of a registry in achieving its aims is determined by the accuracy and completeness of its data. This study identifies marked variability in registry quality and data management practices between countries as well as between subspecialty domains within countries. Few studies have previously assessed data quality across registries, although this is consistent with some previous data comparing medical clinical registries in Australia. ${ }^{17}$ In the current study, data validation processes and data reliability assessments were frequently not done (or at least not reported). This

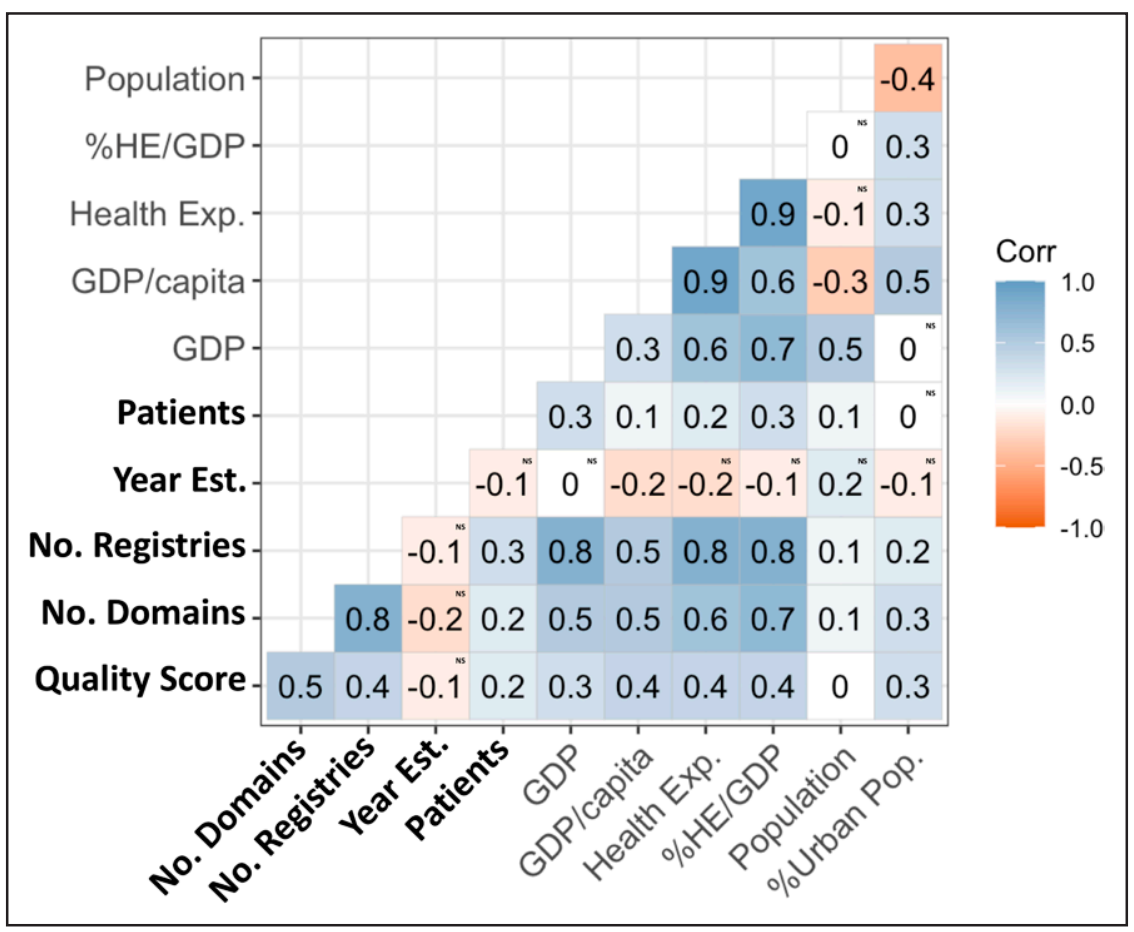

Figure 5. Correlates with registry number, size, and data quality. Correlation matrix demonstrating the correlation coefficients between various variables. A correlation coefficient of $>0.7$ denotes a strong correlation, $0.3-0.7$ a moderate correlation, and $<0.3$ a low correlation. Positive correlations are shown in blue while negative correlations are shown in orange. For example, a country's total number of registries demonstrates a strong positive correlation (0.8) with gross domestic product (GDP), health expenditure (HE), and health expenditure as a percentage of gross domestic product. Registry variables are highlighted in bold, whereas World Bank country variables are not. Exp. indicates expenditure; NS, nonsignificant correlation ( $P>0.05)$; Year Est., year established; Corr, correlation coefficient; and Pop., population. 
is a major concern if significant health policy decisions are to be informed by such registries. Government-mandated patient inclusion and government funding were both associated with higher quality scores. Similarly, registries that could be linked to existing population-based registries (such as in Sweden, ${ }^{9}$ Denmark, ${ }^{11}$ and Fin$\operatorname{land}^{24}$ ) were also associated with higher quality scores. In the absence of a dedicated registry, administrative datasets are sometimes used for similar purposes including for research, governance, or informing health policy. These datasets bring with them various well-described issues including a lack of disease or procedure-specific variables and inaccuracies relating to coding by administrators rather than clinical staff. ${ }^{2}$ However, as demonstrated by several registries included in this review, linkage to these administrative datasets has the potential to enhance the usefulness of registry data. ${ }^{2}$ In our study, disease-based registries had more difficulties with enrollment compared to procedural-based registries and mechanisms to maximize patient-enrollment in these registries, such as the use of opt-out consents and incorporation of registry enrollment processes into the diagnostic or clinical review pathways, should be considered in detail at inception. ${ }^{25}$

Although the overall summary quality score is useful at broadly assessing each registry's quality and data practices, it is important to note that each individual component of this score may be more or less relevant depending on the purpose of the registry. For example, comprehensive enrollment of the at-risk population may be important for health policy but not so important for generating hypotheses for clinical research. Similarly, independence of outcome assessment may be highly important for assessing quality of care and new device safety but less important for monitoring trends in practice or clinical research. Therefore, a clearly defined purpose for a registry is highly important in determining which components of the quality score are worth focusing on during registry development (see Figure II in the Data Supplement). Similarly, if a registry is to be used for an alternate purpose to its original basis, the ability to serve this purpose (eg, clinical research using a quality improvement registry or vice-versa) should be considered with the quality of these individual components in mind.

The rate of registry uptake over the last forty years suggests that national cardiac registries are likely to proliferate going forward. In this setting, careful consideration of both registry aims and data practices is required to enhance their usefulness and avoid misusing the considerable resources required for registry establishment. Managers should aim to ensure data collection and management processes maximize each component of the registry scoring system included in this study. Similarly, funders and policymakers should consider funding and infrastructure models that are most likely to generate high-quality registry data, such as government funding, mandated enrollment, and integration with other population databases. Clearly, resource availability may limit the incorporation of all ideal data practices into registry development. However, the ability of a registry to achieve its aims is largely dependent on the quality of the data, and it should be strongly considered whether the registry will be able to provide quality assurance, inform health policy, facilitate research, and monitor the uptake of new treatments.

While providing the vehicle for reporting on quality of care and outcomes, cardiac registries may also provide the opportunity for improving the quality of care through the conduct of simple comparative effectiveness randomized trials. ${ }^{26,27}$ The SWEDEHEART registry is the global leader in this field and serves as working example of how important clinical questions can be addressed using this platform to resolve equipoise or uncertainty for a fraction of the cost of conventional conduct of randomized trials.

\section{Limitations}

There are several limitations to this review. The search strategy used terms in English, and although we aimed to include non-English studies that were identified during this search through the use of Google translate, there may have been some non-English registries that were not identified. Quality scoring was dependent on the availability of commentary on the specific quality score component in published material from that registry. A proportion of registries did not publish material sufficient to answer all components of this score, especially in the assessment of data reliability, resulting in a not available score (1 point). We attempted to address this by contacting each registry, however among registries that did not respond, this limitation remained. Furthermore, the importance of individual components that comprise the modified Monash University registry grading system differ depending on the purpose of the registry, and the overall score should be interpreted with the registry's intended purpose in mind. Finally, we aimed to include national registries in this review, and there are a large number of both international collaborative registries (such as the European Society of Cardiology registries) and regional registries that were therefore not included. International registries are worthy of special consideration given their large scale and often high-quality data practices (eg, European Society of Cardiology registries), but their inclusion in this study was felt to be outside the scope of the review. These are included in the Data Supplement with reasons for exclusion.

\section{Conclusions}

This review highlights remarkable growth in the use of national cardiac registries across the last few decades. We identified variable quality in processes used to ensure data completeness and accuracy, and only a minority of 
countries had integrated combined registries covering multiple subspecialty domains. Given the multiple benefits, clinicians, funders and health policymakers should be encouraged to focus on the range, quality, and integration of national registries.

\section{ARTICLE INFORMATION}

Received February 15, 2021; accepted May 26, 2021.

\section{Affiliations}

Department of Cardiology, The Royal Melbourne Hospital, Melbourne, Victoria Australia (L.P.D., J.L., L.B., D.E.). Department of Epidemiology and Preventive Medicine, Monash University, Melbourne, Victoria, Australia (L.P.D., S.B., J.L., D.S., S.M.E., C.R.). Department of Cardiology, The Alfred Hospital, Melbourne, Victoria, Australia (L.P.D., D.S.). The Baker Institute, Melbourne, Victoria, Australia (D.S.). Department of Medicine, Melbourne University, Victoria, Australia (L.B., D.E.). Curtin School of Population Health, Curtin University, Perth, Western Australia, Australia (C.R.).

\section{Sources of Funding}

None.

\section{Disclosures}

L.P. Dawson is supported by National Health and Medical Research Council (NHMRC) and National Heart Foundation postgraduate scholarships. Dr Reid is supported by a NHMRC Principal Research Fellowship (reference no. 1136972), Associate Professor Dr Stub's work is supported by a National Heart Foundation Fellowship (reference no. 101908). The other authors report no conflicts.

\section{Supplemental Materials}

Tables I-VI

Figures I-II

References 28-249

\section{REFERENCES}

1. Biswas S, Lefkovits J, Liew D, Gale CP, Reid CM, Stub D. Characteristics of national and major regional percutaneous coronary intervention registries: a structured literature review. Eurolntervention. 2018;14:1112-1120. doi: 10.4244/EIJ-D-18-00434

2. Hickey GL, Grant SW, Cosgriff R, Dimarakis I, Pagano D, Kappetein AP, Bridgewater B. Clinical registries: governance, management, analysis and applications. Eur J Cardiothorac Surg. 2013;44:605-614. doi: 10.1093/ ejcts/ezt018

3. Emilsson L, Lindahl B, Köster M, Lambe M, Ludvigsson JF. Review of 103 Swedish healthcare quality registries. J Intern Med. 2015;277:94-136. doi: 10.1111/joim.12303

4. Cameron PA, Gabbe BJ, Cooper DJ, Walker T, Judson R, McNeil J. A statewide system of trauma care in Victoria: effect on patient survival. Med J Aust. 2008;189:546-550. doi: 10.5694/j.1326-5377.2008.tb02176.x

5. Jakobsen E, Green A, Oesterlind K, Rasmussen TR, lachina M, Palshof T. Nationwide quality improvement in lung cancer care: the role of the Danish lung cancer group and registry. J Thorac Oncol. 2013;8:1238-1247. doi: 10.1097/JTO.0b013e3182a4070f

6. Mallinson EK, Newton KF, Bowen J, Lalloo F, Clancy T, Hill J, Evans DG. The impact of screening and genetic registration on mortality and colorectal cancer incidence in familial adenomatous polyposis. Gut. 2010;59:13781382. doi: 10.1136/gut.2010.212449

7. Wu CK, Juang JJ, Chiang JY, Li YH, Tsai CT, Chiang FT. The Taiwan heart registries: its influence on cardiovascular patient care. J Am Coll Cardiol. 2018;71:1273-1283. doi: 10.1016/j.jacc.2018.02.006

8. Egeland G, Sundvor V, Igland J, Seliussen I, Klakegg Y, Vollset S, Akerkar R, Tell G, Ebbing M. Opportunities for diabetes research using the Norwegian Cardiovascular Disease Registry. Norsk Epidemiologi. 2013;23:95-100. doi: 10.5324/nje.v23i1.1608

9. Jernberg T, Attebring MF, Hambraeus K, Ivert T, James S, Jeppsson A, Lagerqvist B, Lindahl B, Stenestrand U, Wallentin L. The Swedish Websystem for enhancement and development of evidence-based care in heart disease evaluated according to recommended therapies (SWEDEHEART). Heart. 2010;96:1617-1621. doi: 10.1136/hrt.2010.198804
10. Messenger JC, Ho KK, Young CH, Slattery LE, Draoui JC, Curtis JP, Dehmer GJ, Grover FL, Mirro MJ, Reynolds MR, et al; NCDR Science and Quality Oversight Committee Data Quality Workgroup. The National Cardiovascular Data Registry (NCDR) Data Quality Brief: the NCDR Data Quality Program in 2012. J Am Coll Cardiol. 2012;60:1484-1488. doi: 10.1016/j.jacc.2012.07.020

11. Özcan C, Juel K, Flensted Lassen J, von Kappelgaard LM, Mortensen PE, Gislason G. The Danish heart registry. Clin Epidemiol. 2016;8:503-508. doi: 10.2147/CLEP.S99475

12. Freeman JV, Varosy P, Price MJ, Slotwiner D, Kusumoto FM, Rammohan C, Kavinsky CJ, Turi ZG, Akar J, Koutras C, et al. The NCDR left atrial appendage occlusion registry. J Am Coll Cardiol. 2020;75:1503-1518. doi: 10.1016/j.jacc.2019.12.040

13. Holmes DR Jr, Nishimura RA, Grover FL, Brindis RG, Carroll JD, Edwards FH, Peterson ED, Rumsfeld JS, Shahian DM, Thourani VH, et al; STS/ACC TVT Registry. Annual outcomes with transcatheter valve therapy: from the STS/ACC TVT registry. J Am Coll Cardiol. 2015;66:2813-2823. doi: 10.1016/j.jacc.2015.10.021

14. Zhang S, Gaiser S, Kolominsky-Rabas PL; National Leading-Edge Cluster Medical Technologies "Medical Valley EMN. Cardiac implant registries 2006-2016: a systematic review and summary of global experiences. BMJ Open. 2018;8:e019039. doi: 10.1136/bmjopen-2017-019039

15. Zhang S, Kolominsky-Rabas PL. How TAVI registries report clinical outcomes-A systematic review of endpoints based on VARC-2 definitions. PLoS One. 2017;12:e0180815. doi: 10.1371/journal.pone.0180815

16. Moher D, Liberati A, Tetzlaff J, Altman DG; PRISMA Group. Preferred reporting items for systematic reviews and meta-analyses: the PRISMA statement. J Clin Epidemiol. 2009;62:1006-1012. doi: 10.1016/j .jclinepi.2009.06.005

17. Md Emdadul Hoque D, Ruseckaite R, Lorgelly P, McNeil JJ, Evans SM. Crosssectional study of characteristics of clinical registries in Australia: a resource for clinicians and policy makers. Int J Qual Health Care. 2018;30:192-199. doi: 10.1093/intqhc/mzx196

18. WorldBank. World Development Indicators: GDP per capita (current US\$). World Bank National Accounts Data, and OECD National Accounts Data Files. 2020. Accessed April 1, 2021. https://data.worldbank.org/indicator/NY.GDP.PCAP.CD?view=chart

19. Rashid M, Ludman PF, Mamas MA. British Cardiovascular Intervention Society registry framework: a quality improvement initiative on behalf of the National Institute of Cardiovascular Outcomes Research (NICOR). Eur Heart J Qual Care Clin Outcomes. 2019;5:292-297. doi: 10.1093/ehjqcco/qcz023

20. Alger HM, Rutan C, Williams JH $4^{\text {th }}$, WalchokJG, Bolles M, Hall JL, Bradley SM, Elkind MSV, Rodriguez F, Wang TY, et al. American Heart Association COVID-19 CVD registry powered by get with the guidelines. Circ Cardiovasc Qual Outcomes. 2020;13:e006967. doi: 10.1161/CIRCOUTCOMES. 120.006967

21. Resnic FS, Majithia A, Marinac-Dabic D, Robbins S, Ssemaganda H, Hewitt K, Ponirakis A, Loyo-Berrios N, Moussa I, Drozda J, et al. Registrybased prospective, active surveillance of medical-device safety. $N$ Engl J Med. 2017;376:526-535. doi: 10.1056/NEJMoa1516333

22. Dehmer GJ, Jennings J, Madden RA, Malenka DJ, Masoudi FA, McKay CR, Ness DL, Rao SV, Resnic FS, Ring ME, et al. The National Cardiovascular Data Registry Voluntary Public Reporting Program: an interim report from the NCDR Public Reporting Advisory Group. J Am Coll Cardiol. 2016;67:205-215. doi: 10.1016/j.jacc.2015.11.001

23. Wasfy JH, Borden WB, Secemsky EA, McCabe JM, Yeh RW. Public reporting in cardiovascular medicine: accountability, unintended consequences, and promise for improvement. Circulation. 2015;131:1518-1527. doi: 10.1161/CIRCULATIONAHA.114.014118

24. Kiviniemi TO, Pietilä A, Gunn JM, Aittokallio JM, Mähönen MS, Salomaa VV, Niiranen TJ. Trends in rates, patient selection and prognosis of coronary revascularisations in Finland between 1994 and 2013: the CVDR. Eurolntervention. 2016;12:1117-1125. doi: 10.4244/EIJV12I9A183

25. McNeil JJ, Evans SM, Johnson NP, Cameron PA. Clinical-quality registries: their role in quality improvement. Med J Aust. 2010;192:244-245. doi: 10.5694/j.1326-5377.2010.tb03499.x

26. Lauer MS, D'Agostino RB Sr. The randomized registry trial-the next disruptive technology in clinical research? N Engl J Med. 2013;369:1579-1581. doi: 10.1056/NEJMp1310102

27. Fröbert O, Lagerqvist B, Olivecrona GK, Omerovic E, Gudnason T, Maeng M, Aasa M, Angerås O, Calais F, Danielewicz M, et al; TASTE Trial. Thrombus aspiration during ST-segment elevation myocardial infarction. N Engl J Med. 2013;369:1587-1597. doi: 10.1056/NEJMoa1308789 